\title{
Nuclear data analyses for improving the safety of advanced lead-cooled reactors
}

\author{
Pablo Romojaro ${ }^{1, *}$, Francisco Álvarez-Velarde ${ }^{1}$, and Nuria García-Herranz ${ }^{2}$ \\ ${ }^{1}$ CIEMAT, Energy Department, Avenida Complutense 40, 28040, Spain \\ ${ }^{2}$ UPM, Energy Engineering Department, Calle José Gutiérrez Abascal 2, 28006, Spain
}

\begin{abstract}
A target accuracy assessment of the effective neutron multiplication factor, $k_{\text {eff }}$, for MYRRHA (Multi-purpose hYbrid Research Reactor for High-tech Applications) lead-bismuth cooled fast reactor has been performed with JEFF-3.3 and ENDF/B-VIII.0 state-of-the-art nuclear data libraries and the SUMMON system. Uncertainties in $k_{\text {eff }}$ due to uncertainties in nuclear data have been assessed against the target accuracies provided by SG-26 of the WPEC of OECD/NEA in 2008 for LFR. Results show that $k_{\text {eff }}$ target accuracy is still exceeded by more than a factor of two using the latest nuclear data evaluations released in 2018. Consequently, nuclear data assimilation has been carried out using criticality experiments from the International Criticality Safety Benchmark Evaluation Project that are representative of MYRRHA. The results from this work show that the level of accuracy needed in nuclear data cannot be obtained using only differential experiments, but the combination of experimental covariance data and integral experiments together with Generalised Least Squares technique can provide adjusted nuclear data capable of predicting reactor properties with lower uncertainty and consistent with differential data.
\end{abstract}

\section{Introduction}

The Lead-cooled Fast Reactor (LFR) is one of the three technologies selected by the Sustainable Nuclear Energy Technology Platform (SNETP) [1] that can meet future European energy needs. Significant efforts are being made by researchers and industry to overcome the main drawbacks for the industrial deployment of LFR, which are the lack of operational experience and the impact of uncertainties in the reactor design, operation and safety assessment.

In nuclear reactor design the uncertainties mainly come from material properties, fabrication tolerances, operative conditions, simulation tools and nuclear data. However, even though the uncertainty in nuclear data is one of the most important sources of uncertainty in reactor design and reactor physics simulations [2], significant gaps between the uncertainties and the target accuracies have been systematically shown in the past [3]. Meeting the target accuracy is required not only to achieve the requested level of safety for this technology, but also to minimize the increase in the costs due to additional security measures.

\footnotetext{
*Corresponding author: pablo.romojaro@ciemat.es
} 
In this paper, a target accuracy assessment of the effective neutron multiplication factor, $k_{\text {eff, }}$, for MYRRHA (Multi-purpose hYbrid Research Reactor for High-tech Applications) [4] lead-bismuth cooled fast reactor is performed with the JEFF-3.3 [5] and ENDF/B-VIII.0 [6] state-of-the-art nuclear data libraries and the SUMMON system [7]. Uncertainty quantification (UQ) analyses have already been carried out for previous MYRRHA designs [8-10] or with older nuclear data evaluations [11]; nevertheless, since JEFF-3.3 and ENDF/B.VIII.0 were released, an updated analysis was required in order to take into account the changes in the new nuclear data libraries and their impact in the criticality safety coefficients.

Furthermore, data assimilation for the main isotopes contributing to the uncertainty has been performed using critical mass experiments from the International Criticality Safety Benchmark Evaluation Project (ICSBEP) [12] that are representative of MYRRHA, in order to obtain adjusted nuclear data with constrained uncertainties and to reduce the uncertainty in the criticality parameter.

\section{SUMMON system}

The Sensitivity and Uncertainty Methodology for MONtecarlo codes (SUMMON) system [7, 13] has been conceived by CIEMAT as a tool to perform complete automated sensitivity and uncertainty analyses of the most relevant criticality safety parameters of detailed complex reactor designs from the neutronic point of view, i.e., $k_{\text {eff, }} \beta_{\text {eff }}, \Lambda_{\text {eff }}$ and reactivity coefficients, using state-of-the-art nuclear data libraries and covariances.

A detailed description of SUMMON, including the methodologies used to calculate the sensitivity coefficients and perform the uncertainty quantification analyses, can be found in Ref. [7, 13].

Recently, the Data Assimilation With summoN (DAWN) module has been developed to perform data assimilation using integral experiments from public databases, with the aim of providing adjusted nuclear data, not only capable of predicting reactor properties within the target design accuracy, but also statistically consistent with the various differential measurements.

DAWN is based on the generalised least squares technique [14], in which the evaluation of the posterior expectation of the cross sections, $\sigma^{\prime}$, and covariances, $M_{\sigma}^{\prime}$, is done by finding the minimum of the following function:

$$
\chi^{2}=\left(\frac{\sigma^{\prime}-\sigma}{\sigma}\right)^{T} M_{\sigma}^{-1}\left(\frac{\sigma^{\prime}-\sigma}{\sigma}\right)+\left(\frac{E-C}{C}\right)^{T} M_{E C}^{-1}\left(\frac{E-C}{C}\right)
$$

where $\sigma$ and $M_{\sigma}$ are the a priori expectation and covariance matrix of the cross sections, $E$ is the experimental value of the measured integral parameter, $C$ is the a priori calculated value of the integral parameter and $M_{E C}$ is the relative integral parameter covariance matrix.

The cross sections modifications that minimize the $\chi^{2}$ thanks to the adjustment are:

$$
\left(\frac{\sigma^{\prime}-\sigma}{\sigma}\right)=M_{\sigma} S^{T} G^{-1}\left(\frac{E-C}{C}\right)
$$

and the associated a posteriori covariance matrix is:

$$
M_{\sigma}^{\prime}=M_{\sigma}-M_{\sigma} S^{T} G^{-1} S M_{\sigma}=M_{\sigma}\left(I-S^{T} G^{-1} S M_{\sigma}\right)
$$

being $S$ the sensitivities of nuclear data to the integral parameter and $G$ the relative total integral covariance matrix. 


\section{Sensitivity and uncertainty analyses}

MYRRHA's updated core design is described in detail in Ref. [4]. For this work a simplified model [15], homogenised on fuel assembly level, of the critical core configuration in nominal conditions at Beginning of Life (BoL) has been used. The layout of the core is shown in Fig. 1.
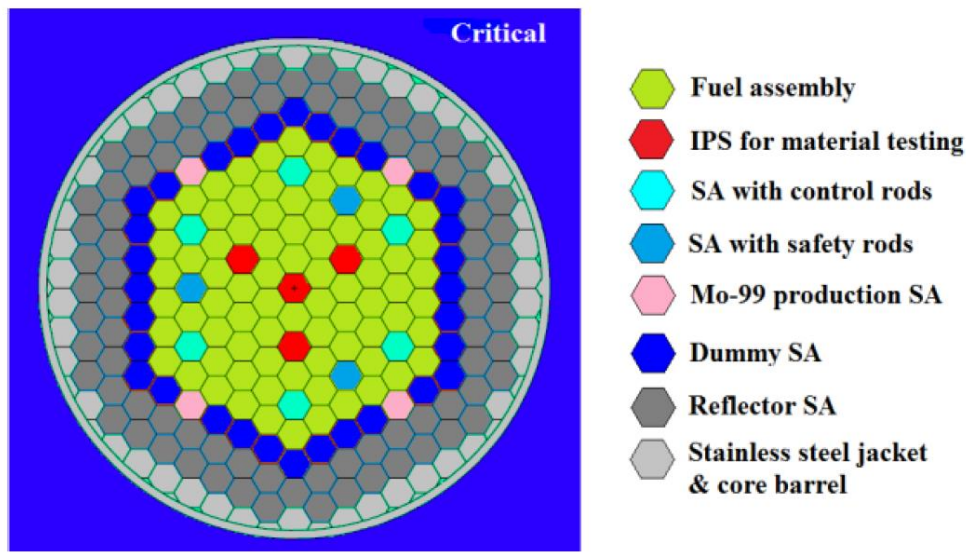

Fig. 1. Critical MYRRHA core layout.

Sensitivity calculations were performed in order to obtain the sensitivity coefficients for uncertainty propagation and to identify the most important nuclear data for neutron induced reactions in MYRRHA. A comparison between the Integrated Sensitivity Coefficients (ISC) obtained using JEFF-3.3 and ENDF/B-VIII.0 nuclear data libraries of the most relevant reactions for $k_{\text {eff }}$ is shown in Table 1 . It can be seen that the sensitivity coefficients obtained with both libraries are practically the same, therefore for the uncertainty quantification, the uncertainties in nuclear data have been propagated using only the sensitivities derived with JEFF-3.3 library.

Table 1. ISC of the top 5 reactions for MYRRHA using JEFF-3.3 and ENDF/B-VIII.0 libraries.

\begin{tabular}{|c|c|c|c|}
\hline \multicolumn{4}{|c|}{ MYRRHA - ISC (\%/\%) } \\
\hline \multicolumn{2}{|c|}{ Quantity } & JEFF-3.3 & ENDF/B-VIII.0 \\
\hline${ }^{239} \mathrm{Pu}$ & $v$ & 0.696 & 0.698 \\
\hline${ }^{239} \mathrm{Pu}$ & $(\mathrm{n}, \mathrm{f})$ & 0.482 & 0.486 \\
\hline${ }^{238} \mathrm{U}$ & $(\mathrm{n}, \gamma)$ & -0.112 & -0.115 \\
\hline${ }^{240} \mathrm{Pu}$ & $v$ & 0.081 & 0.080 \\
\hline${ }^{239} \mathrm{Pu}$ & $(\mathrm{n}, \gamma)$ & -0.053 & -0.056 \\
\hline
\end{tabular}

Using the "Sandwich Rule" of the "Propagation of Moments" method [7], the uncertainties were propagated with both JEFF-3.3 and ENDF/B-VIII.0 covariance data. The top 10 contributors, as well as the total uncertainty in $k_{\text {eff }}$ due to these contributors, are presented in Table 2. A good agreement can be observed in the total $k_{\text {eff }}$ uncertainty derived by JEFF-3.3 and ENDF/B-VIII.0, even though the contributors differ. This can be attributed to differences in the evaluations, such as the case of ${ }^{240} \mathrm{Pu}(\mathrm{n}, \mathrm{f})$, to missing correlations, such as ${ }^{240} \mathrm{Pu}(\mathrm{n} . \mathrm{f})-{ }^{240} \mathrm{Pu}(\mathrm{n}, \gamma)$ in ENDF/B-VIII.0, and to missing covariance evaluations, such as ${ }^{209} \mathrm{Bi}(\mathrm{n}, \mathrm{el}$.) in JEFF-3.3. 
Table 2. UQ for MYRRHA using different covariance evaluations.

MYRRHA

\begin{tabular}{|c|c|c|c|c|c|c|c|c|c|}
\hline \multicolumn{5}{|c|}{ JEFF-3.3 } & \multicolumn{5}{|c|}{ ENDF/B-VIII.0 } \\
\hline & \multicolumn{2}{|c|}{ Quantity } & & $\Delta k_{\text {eff }} / k_{\text {eff }}(\%)$ & \multicolumn{4}{|c|}{ Quantity } & \multirow{2}{*}{$\frac{\Delta \boldsymbol{k}_{\text {eff }} / \boldsymbol{k}_{\text {eff }}(\%)}{0.550}$} \\
\hline${ }^{240} \mathrm{Pu}$ & $(\mathrm{n}, \mathrm{f})$ & ${ }^{240} \mathrm{Pu}$ & $(\mathrm{n}, \mathrm{f})$ & 0.543 & ${ }^{239} \mathrm{Pu}$ & $(\mathrm{n}, \mathrm{f})$ & ${ }^{239} \mathrm{Pu}$ & $(\mathrm{n}, \mathrm{f})$ & \\
\hline${ }^{240} \mathrm{Pu}$ & $(\mathrm{n}, \mathrm{f})$ & ${ }^{240} \mathrm{Pu}$ & $(\mathrm{n}, \gamma)$ & -0.420 & ${ }^{239} \mathrm{Pu}$ & $(\mathrm{n}, \gamma)$ & ${ }^{239} \mathrm{Pu}$ & $(\mathrm{n}, \gamma)$ & 0.227 \\
\hline${ }^{239} \mathrm{Pu}$ & $v$ & ${ }^{239} \mathrm{Pu}$ & $v$ & 0.321 & ${ }^{209} \mathrm{Bi}$ & $(\mathrm{n}, \mathrm{el})$ & ${ }^{209} \mathrm{Bi}$ & $(\mathrm{n}, \mathrm{el})$ & 0.222 \\
\hline${ }^{239} \mathrm{Pu}$ & $(\mathrm{n}, \mathrm{f})$ & ${ }^{239} \mathrm{Pu}$ & $(\mathrm{n}, \mathrm{f})$ & 0.295 & ${ }^{239} \mathrm{Pu}$ & $v_{p}$ & ${ }^{239} \mathrm{Pu}$ & $v_{p}$ & 0.187 \\
\hline${ }^{239} \mathrm{Pu}$ & $\chi$ & ${ }^{239} \mathrm{Pu}$ & $\chi$ & 0.261 & ${ }^{238} \mathrm{U}$ & $(\mathrm{n}, \gamma)$ & ${ }^{238} \mathrm{U}$ & $(\mathrm{n}, \gamma)$ & 0.139 \\
\hline${ }^{240} \mathrm{Pu}$ & $(\mathrm{n}, \gamma)$ & ${ }^{240} \mathrm{Pu}$ & $(\mathrm{n}, \gamma)$ & 0.197 & ${ }^{239} \mathrm{Pu}$ & $\chi$ & ${ }^{239} \mathrm{Pu}$ & $\chi$ & 0.128 \\
\hline${ }^{239} \mathrm{Pu}$ & $(\mathrm{n}, \mathrm{f})$ & ${ }^{239} \mathrm{Pu}$ & $(\mathrm{n}, \gamma)$ & 0.174 & ${ }^{238} \mathrm{U}$ & $(\mathrm{n}, \mathrm{in})$ & ${ }^{238} \mathrm{U}$ & $(\mathrm{n}, \mathrm{f})$ & -0.095 \\
\hline${ }^{238} \mathrm{U}$ & $(\mathrm{n}, \gamma)$ & ${ }^{238} \mathrm{U}$ & $(\mathrm{n}, \gamma)$ & 0.167 & ${ }^{240} \mathrm{Pu}$ & $(\mathrm{n}, \gamma)$ & ${ }^{240} \mathrm{Pu}$ & $(\mathrm{n}, \gamma)$ & 0.091 \\
\hline${ }^{239} \mathrm{Pu}$ & $(\mathrm{n}, \gamma)$ & ${ }^{23} \mathrm{Pu}$ & $(\mathrm{n}, \gamma)$ & 0.151 & ${ }^{238} \mathrm{U}$ & $v_{p}$ & ${ }^{238} \mathrm{U}$ & $v_{\mathrm{p}}$ & 0.087 \\
\hline${ }^{238} \mathrm{U}$ & $(\mathrm{n}$, in $)$ & ${ }^{238} \mathrm{U}$ & $(\mathrm{n}, \mathrm{f})$ & -0.138 & ${ }^{238} \mathrm{U}$ & $(\mathrm{n}, \mathrm{in})$ & ${ }^{238} \mathrm{U}$ & $(\mathrm{n}, \gamma)$ & 0.081 \\
\hline \multicolumn{4}{|c|}{ Uncertainty in } & 0.691 & \multicolumn{4}{|c|}{ Uncertainty in $\boldsymbol{k}_{\text {eff }}$} & 0.698 \\
\hline
\end{tabular}

Design target accuracies for a wide range of innovative systems were provided in 2008 by the SG-26 of the Working Party on International Nuclear Data Evaluation Co-operation of OECD/NEA [16]. For fast reactors at BoL, a target accuracy of $300 \mathrm{pcm}$ in the effective neutron multiplication factor was specified. Nearly $700 \mathrm{pcm}$ of uncertainty in $k_{\text {eff }}$ have been obtained in the UQ using the state-of-the-art JEFF-3.3 and ENDF/B-VIII.0 libraries; therefore, the design target accuracy is still exceeded by more than a factor of two for the considered modern nuclear data evaluations.

\section{Data assimilation}

In order to constrain the uncertainties, an assimilation on the main contributors to the uncertainty in $k_{\text {eff, }}$, i.e., ${ }^{240} \mathrm{Pu},{ }^{239} \mathrm{Pu}$ and ${ }^{238} \mathrm{U}$ nuclear data, was performed using only JEFF-3.3 cross sections and covariances as a prior.

However, before nuclear data can be adjusted, the first step is to select a comprehensive set of complementary experiments, representative of the target application. In practice, this means calculating correlations among the reactor concept and experiments to determine how representative is the latter of the former. This was done by means of the representativity factor, $f_{R E}$ :

$$
f_{R E}=\frac{S_{R}^{T} M_{\sigma} S_{E}}{\sqrt{\left(S_{R}^{T} M_{\sigma} S_{R}\right)\left(S_{E}^{T} M_{\sigma} S_{E}\right)}}
$$

where $S_{R}$ is the sensitivity vector for the targeted reactor and $S_{E}$ is the sensitivity vector for the experiment. The closer $f_{R E}$ is to one, the more similarity exists between the reactor and the experiment.

Publicly available critical mass experiments from the ICSBEP database were assessed and a set of three experiments that provide information on separated individual physics effects related to the isotopes with highest sensitivity were chosen to perform data assimilation. However, correlation coefficient data of uncertainties in criticality cases only exist for a limited number of integral experiments. In particular, only for the ones derived from IPPE and from ANL for the ZPR/ZPPR values are publicly available in the ICSBEP database [17]. Therefore, if all three experiments were used in the data adjustment without taking into account correlations, possible compensation effects may occur. Consequently, three separate data adjustments have been carried out. The experiments selected were: 
- JEZEBEL (PMF001) [18]: bare sphere of ${ }^{239} \mathrm{Pu}$ metal, sensitive to ${ }^{239} \mathrm{Pu}$;

- ${ }^{240} \mathrm{Pu}$ JEZEBEL (PMF002) [19]: bare sphere of ${ }^{239} \mathrm{Pu}$ metal with 20.1 at $\%{ }^{240} \mathrm{Pu}$, sensitive to ${ }^{240} \mathrm{Pu}$;

- PU-MET-FAST-006 (PFM006) [20]: plutonium sphere reflected by uranium, sensitive to ${ }^{238} \mathrm{U}$.

In Table 3, the representativity factors between MYRRHA and the three experiments and among the experiments themselves are shown. While the most representative experiment is PMF002, a close similarity between the experiments is observed, due to all of them employing plutonium as fissile material and having similar energy spectrums.

Table 3. Representativity factor of the experiments used in the assimilation.

\begin{tabular}{c|cccc}
$\boldsymbol{f}_{\boldsymbol{R} E}$ & MYRRHA & PMF001 & PMF002 & PMF006 \\
\hline MYRRHA & 1.00 & 0.71 & 0.88 & 0.52 \\
PMF001 & 0.71 & 1.00 & 0.78 & 0.98 \\
PMF002 & 0.88 & 0.78 & 1.00 & 0.75 \\
PMF006 & 0.52 & 0.98 & 0.75 & 1.00
\end{tabular}

Once the assimilation was performed, the posterior covariance matrices and original sensitivity coefficients were used to propagate the uncertainties. The consistency of the assimilation was assessed against experimental data. An example of the consistency checks is given in Fig. 2, where the prior and posterior ${ }^{240} \mathrm{Pu}(\mathrm{n}, \mathrm{f})$ cross sections after the assimilation with PMF002 integral experiment are represented. It can be seen that the central value of the cross section has been modified in the fast energy range and is compatible with the various experimental differential data.

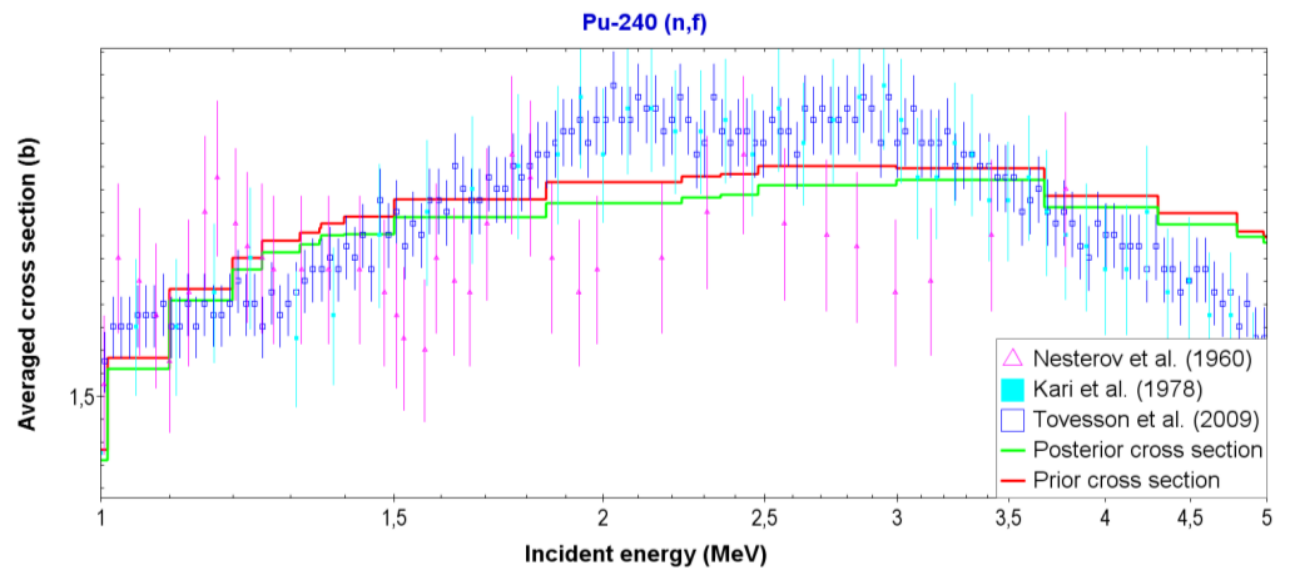

Fig. 2. ${ }^{240} \mathrm{Pu}(\mathrm{n}, \mathrm{f})$ prior and posterior cross sections compared with experimental data.

The results from the UQ using the posterior covariance matrices are presented in Table 4. The highest reduction in MYRRHA $k_{\text {eff }}$ uncertainty is given by the assimilation of PMF002 experiment (the most sensible to ${ }^{240} \mathrm{Pu}$ and most representative of MYRRHA) due to the substantial reduction in the uncertainty of the two major contributors, i.e., ${ }^{240} \mathrm{Pu}(\mathrm{n}, \mathrm{f})$ and ${ }^{240} \mathrm{Pu}(\mathrm{n}, \mathrm{f})-{ }^{240} \mathrm{Pu}(\mathrm{n}, \gamma)$. Furthermore, new strong negative cross-correlations between isotopes and reactions appear after the adjustment, such as ${ }^{239} \mathrm{Pu} v_{\mathrm{p}^{-}}{ }^{240} \mathrm{Pu}(\mathrm{n}, \mathrm{f})$, which are the main responsible for the significant reductions in the total uncertainty using PMF001 and PMF002 integral experiments. On the other hand, the use of the PMF006 for the assimilation produces negligible adjustments that have insignificant impact when the uncertainties are propagated. This can be attributed to the low similarity of PMF006 with MYRRHA, in spite of the high sensitivity of this experiment to ${ }^{238} \mathrm{U}$ in the fast energy range. 
Table 4. UQ for MYRRHA using adjusted covariance matrices.

MYRRHA

\begin{tabular}{|c|c|c|c|c|c|c|c|c|c|c|c|c|c|}
\hline \multicolumn{5}{|c|}{ Adjusted JEFF-3.3 with PMF001 } & \multicolumn{4}{|c|}{ Adjusted JEFF-3.3 with PMF002 } & \multicolumn{5}{|c|}{ Adjusted JEFF-3.3 with PMF006 } \\
\hline \multicolumn{4}{|c|}{ Quantity } & \multirow{2}{*}{$\begin{array}{c}\begin{array}{c}\Delta \boldsymbol{k}_{\text {eff }} / \boldsymbol{k}_{\text {eff }} \\
\mathbf{( \% )}\end{array} \\
0.525\end{array}$} & \multicolumn{3}{|c|}{ Quantity } & \multirow{2}{*}{$\begin{array}{c}\begin{array}{c}\Delta \boldsymbol{k}_{\text {eff }} / \boldsymbol{k}_{\text {eff }} \\
\mathbf{( \% )}\end{array} \\
0.333\end{array}$} & \multicolumn{4}{|c|}{ Quantity } & \multirow{2}{*}{$\begin{array}{c}\begin{array}{c}\Delta \boldsymbol{k}_{e f f} / \boldsymbol{k}_{\text {eff }} \\
(\%)\end{array} \\
0.539\end{array}$} \\
\hline${ }^{240} \mathrm{Pu}$ & $(\mathrm{n}, \mathrm{f})$ & ${ }^{240} \mathrm{Pu}$ & $(\mathrm{n}, \mathrm{f})$ & & $\mathrm{Pu} \quad(\mathrm{n}, \mathrm{f})$ & ${ }^{0} \mathrm{Pu}$ & $(\mathrm{n}, \mathrm{f})$ & & ${ }^{\circ} \mathrm{Pu}$ & $(\mathrm{n}, \mathrm{f})$ & ${ }^{0} \mathrm{Pu}$ & $(\mathrm{n}, \mathrm{f})$ & \\
\hline${ }^{240} \mathrm{Pu}$ & $(\mathrm{n}, \mathrm{f})$ & ${ }^{240} \mathrm{Pu}$ & $(\mathrm{n}, \gamma)$ & -0.405 & ${ }^{239} \mathrm{Pu} \quad \chi$ & ${ }^{240} \mathrm{Pu}$ & $(\mathrm{n}, \mathrm{f})$ & -0.314 & ${ }^{240} \mathrm{Pu}$ & $(\mathrm{n}, \mathrm{f})$ & ${ }^{240} \mathrm{Pu}$ & $(\mathrm{n}, \gamma)$ & -0.416 \\
\hline${ }^{239} \mathrm{Pu}$ & $(n, f)$ & ${ }^{239} \mathrm{Pu}$ & $(\mathrm{n}, \mathrm{f})$ & 0.292 & ${ }^{239} \mathrm{Pu} \quad v_{\mathrm{p}}$ & ${ }^{239} \mathrm{Pu}$ & $v_{p}$ & 0.303 & ${ }^{239} \mathrm{Pu}$ & $v_{\mathrm{p}}$ & ${ }^{239} \mathrm{Pu}$ & $v_{p}$ & 300 \\
\hline${ }^{239} \mathrm{Pu}$ & $v_{\mathrm{p}}$ & ${ }^{239} \mathrm{Pu}$ & $v_{\mathrm{p}}$ & 0.259 & ${ }^{239} \mathrm{Pu} \quad v_{\mathrm{p}}$ & ${ }^{240} \mathrm{Pu}$ & $(\mathrm{n}, \mathrm{f})$ & -0.302 & ${ }^{239} \mathrm{Pu}$ & $(\mathrm{n}, \mathrm{f})$ & ${ }^{239} \mathrm{Pu}$ & $(\mathrm{n}, \mathrm{f})$ & 0.291 \\
\hline${ }^{239} \mathrm{Pu}$ & $v_{\mathrm{p}}$ & ${ }^{239} \mathrm{Pu}$ & $\chi$ & -0.259 & ${ }^{239} \mathrm{Pu} \quad(\mathrm{n}, \mathrm{f})$ & ${ }^{239} \mathrm{Pu}$ & $(\mathrm{n}, \mathrm{f})$ & 0.294 & ${ }^{239} \mathrm{Pu}$ & $\chi$ & ${ }^{239} \mathrm{Pu}$ & $\chi$ & 0.229 \\
\hline${ }^{239} \mathrm{Pu}$ & $v_{p}$ & ${ }^{240} \mathrm{Pu}$ & $(\mathrm{n}, \mathrm{f})$ & -0.231 & ${ }^{240} \mathrm{Pu} \quad(\mathrm{n}, \mathrm{f})$ & ${ }^{240} \mathrm{Pu}$ & $(\mathrm{n}, \gamma)$ & -0.254 & ${ }^{240} \mathrm{Pu}$ & $(\mathrm{n}, \gamma)$ & ${ }^{240} \mathrm{Pu}$ & $(\mathrm{n}, \gamma)$ & 0.196 \\
\hline${ }^{239} \mathrm{Pu}$ & $\chi$ & ${ }^{240} \mathrm{Pu}$ & $(\mathrm{n}, \mathrm{f})$ & -0.223 & ${ }^{239} \mathrm{Pu} \quad \chi$ & ${ }^{239} \mathrm{Pu}$ & $\chi$ & 0.234 & ${ }^{239} \mathrm{Pu}$ & $v_{p}$ & ${ }^{239} \mathrm{Pu}$ & $\chi$ & -0.169 \\
\hline${ }^{239} \mathrm{Pu}$ & $\chi$ & ${ }^{239} \mathrm{Pu}$ & $\chi$ & 0.193 & ${ }^{239} \mathrm{Pu} \quad(\mathrm{n}, \mathrm{f})$ & ${ }^{239} \mathrm{Pu}$ & $(\mathrm{n}, \gamma)$ & 0.173 & ${ }^{239} \mathrm{Pu}$ & $(\mathrm{n}, \mathrm{f})$ & ${ }^{239} \mathrm{Pu}$ & $(\mathrm{n}, \gamma)$ & 0.169 \\
\hline${ }^{240} \mathrm{Pu}$ & $(\mathrm{n}, \gamma)$ & ${ }^{240} \mathrm{Pu}$ & $(\mathrm{n}, \gamma)$ & 0.192 & ${ }^{239} \mathrm{Pu} \quad \chi$ & ${ }^{240} \mathrm{Pu}$ & $(\mathrm{n}, \gamma)$ & 0.173 & ${ }^{238} \mathrm{U}$ & $(\mathrm{n}, \gamma)$ & ${ }^{238} \mathrm{U}$ & $(\mathrm{n}, \gamma)$ & 0.167 \\
\hline${ }^{239} \mathrm{Pu}$ & $(\mathrm{n}, \mathrm{f})$ & ${ }^{239} \mathrm{Pu}$ & $(\mathrm{n}, \gamma)$ & 0.173 & ${ }^{238} \mathrm{U} \quad(\mathrm{n}, \gamma)$ & ${ }^{238} \mathrm{U}$ & $(\mathrm{n}, \gamma)$ & 0.167 & ${ }^{239} \mathrm{Pu}$ & $(\mathrm{n}, \gamma)$ & ${ }^{239} \mathrm{Pu}$ & $(\mathrm{n}, \gamma)$ & 0.150 \\
\hline \multicolumn{4}{|c|}{ Uncertainty in $\boldsymbol{k}_{\text {eff }}$} & 0.444 & \multicolumn{3}{|c|}{ Uncertainty in $\boldsymbol{k}_{\text {eff }}$} & 0.422 & \multicolumn{4}{|c|}{ Uncertainty in $\boldsymbol{k}_{\text {eff }}$} & 0.658 \\
\hline
\end{tabular}

\section{Conclusions}

A target accuracy assessment of the effective neutron multiplication factor for MYRRHA innovative lead-bismuth cooled fast reactor has been carried out using the JEFF-3.3 and ENDF/B-VIII.0 state-of-the-art nuclear data libraries and the SUMMON system. Similar sensitivities and total $k_{\text {eff }}$ uncertainty have been derived using both libraries, however, the contributors to the uncertainty significantly differ. This have been attributed to differences in the evaluations, to missing correlations and to missing covariance evaluations. Moreover, the uncertainties in $k_{\text {eff }}$ due to uncertainties in nuclear data have been assessed against the target accuracies provided by SG-26 of the WPEC of OECD/NEA in 2008 for LFR. Results show that $k_{\text {eff }}$ target accuracy is still exceeded by more than a factor of two using the latest nuclear data evaluations released in 2018.

In order to constrain the uncertainties in $k_{\text {eff, }}$ an assimilation on the main contributors to the uncertainty has been performed using only JEFF-3.3 nuclear data as a prior and publicly available critical mass experiments from the International Criticality Safety Benchmark Evaluation Project. The consistency of the nuclear data adjustment has been checked against differential experimental data and good agreement has been found. A significant reduction in $k_{\text {eff }}$ uncertainty has been obtained using the experiments most representative of MYRRHA, due to the reduction in the uncertainty of the major contributors and to the presence a posteriori of strong cross-correlations between isotopes and reactions that did not exist a priori. Results show that a reduction of nearly $300 \mathrm{pcm}$ can be achieved performing an assimilation with the most sensitive experiment to the major contributor to the uncertainty, proving that the combination of experimental covariance data and integral experiments together with Generalised Least Squares technique, can provide adjusted nuclear data capable of predicting reactor properties with lower uncertainty and consistent with differential data.

\section{References}

1. Sustainable Nuclear Energy Technology Platform. http://www.snetp.eu

2. I. Kodeli, Report OECD/NEA (2007)

3. H. Harada, A. Plompen, NEA/NSC/WPEC/DOC(2014)446 (2014)

4. G. Van den Eynde et al., J. Nucl. Sci. Technol. 52, 1053 (2015)

5. A. Plompen, OECD/NEA JEF/DOC-1864 (2017) 
6. D.A. Brown et al., Nucl. Data Sheets 148, 1-142 (2018)

7. P. Romojaro et al., $M \& C 2017$ (2017)

8. C.J. Díez et al., Nucl. Data Sheets 118, 526-518 (2014)

9. A. Stankovskiy et al., Nucl. Data Sheets 118, 513-515 (2014)

10. T. Sugawara et al., Ann. Nucl. Energy 38, 1098-1108 (2011)

11. P. Romojaro, Ann. Nucl. Energy 101, 330-338 (2017)

12. OECD/NEA, NEA/NSC/DOC(95)03/I (2016)

13. P. Romojaro et al., PHYSOR 2018 (2018)

14. C. De Saint Jean et al., OECD/NEA NEA/NSC/WPEC/DOC(2010)429 (2011)

15. A. Stankovskiy et al., SCK·CEN/4463803 (2014)

16. M. Salvatores et al., OECD/NEA NEA/WPEC-26 (2008)

17. OECD/NEA, NEA/NSC/DOC(95)03/II (2016)

18. OECD/NEA, NEA/NSC/DOC(95)03/I Volume I PU-MET-FAST-001 (2016)

19. OECD/NEA, NEA/NSC/DOC(95)03/I Volume I PU-MET-FAST-002 (2016)

20. OECD/NEA, NEA/NSC/DOC(95)03/I Volume I PU-MET-FAST-006 (2016) 